DOI: 10.1002/ ((please add manuscript number))

Article type: Full Paper

\title{
3D GaN fins as a versatile platform for a-plane based devices
}

Jana Hartmann*, Irene Manglano Clavero, Lars Nicolai, Christoph Margenfeld, Hendrik Spende, Johannes Ledig, Hao Zhou, Frederik Steib, Angelina Jaros, Adrian Avramescu, Martin Strassburg, Achim Trampert, Hergo-Heinrich Wehmann, Hans-Jürgen Lugauer, Tobias Voss, Andreas Waag

Dr. J. Hartmann, I. Manglano Clavero, C. Margenfeld, H. Spende, H. Zhou, F. Steib, A. Jaros, apl. Prof. H.-H. Wehmann, Prof. T. Voss, Prof. A. Waag

Institut für Halbleitertechnik and Laboratory for Emerging Nanometrology, Technische Universität Braunschweig, Hans-Sommer-Str. 66, 38106 Braunschweig, Germany

E-mail: Jana.Hartmann@tu-bs.de

J. Ledig

Physikalisch-Technische Bundesanstalt (PTB), Bundesallee 100, 38116 Braunschweig, Germany

L. Nicolai, Dr. A. Trampert

Paul-Drude-Institut für Festkörperelektronik, Hausvogteiplatz 5-7, 10117 Berlin, Germany

Dr. A. Avramescu, Dr. M. Strassburg, Dr. H.-J. Lugauer

OSRAM Opto Semiconductors GmbH, Leibnizstraße 4, 93055 Regensburg, Germany

Keywords: GaN fins, threading dislocation density, a-plane sidewalls, selective area MOVPE, marker layers

GaN fins on GaN-on-sapphire templates are fabricated by continuous mode selective area metalorganic vapor phase epitaxy. The fins exhibit high aspect ratios and smooth nonpolar aplane sidewalls with an ultra-low threading dislocation density of a few $10^{5} \mathrm{~cm}^{-2}$ making them ideally suited for optoelectronic to electronic applications. A detailed analysis of the inner structure of GaN fins is provided by the help of marker layer experiments and correlation of results from fins fabricated under different growth conditions, leading to the development of a growth model to explain the final geometry and optical as well as electrical properties of these high aspect ratio fins. Distinctly different material properties for the central and outer parts of the fins are detected. Whereas the outer sidewalls represent high quality GaN surfaces with 


\section{WILEY-VCH}

very low defect densities, a strong quenching of near band edge emission (NBE) in the central part of the fins is accompanied by heavy compensation of free electrons. A possible explanation is the incorporation of excessive point defects, like intrinsic defects or carbon impurity. The sidewall regions, however, prove to be highly suitable for device applications due to their strong NBE emission, low dislocation density and high free carrier concentration.

\section{Introduction}

Today, GaN based electronic and optoelectronic devices have already reached a mature development stage. However, as of now, GaN technology is still relying on foreign substrates, mostly sapphire ${ }^{1,2,3}$ and silicon ${ }^{4}$ but more recently also oxide materials such as $\operatorname{LSAT}^{5}$ or spinel and related compounds ${ }^{6}$. These foreign substrates generally lead to high threading dislocation densities in the range of $10^{8} \mathrm{~cm}^{-2}$ in planar $\mathrm{GaN}$ thin films. ${ }^{7,8}$ Initially it has been very surprising that high efficiency light emitting diodes (LEDs) based on heterostructures can be realized under these conditions. Blue and green emitting LEDs have been proven to be very insensitive to defect densities, which has been explained by localisation mechanisms in their active region keeping free charge carriers away from extended defects. ${ }^{9}$ For other wavelength ranges, in particular for the UV spectral range, LEDs seem to be much more sensitive to defects. This is one of the reasons why the efficiency of UV LEDs is more than one order of magnitude lower than in the blue and green spectral range. ${ }^{10,11}$ Low defect density platforms are urgently needed to further develop UV LED technology. While bulk GaN substrates provide extremely low densities of extended defects ${ }^{12}$, high cost and the lack of large substrate diameters hinder their application in cost-sensitive applications like LED manufacturing. ${ }^{13}$ Due to mismatch in lattice parameters and thermal expansion coefficients, foreign substrates inevitably introduce not only extended lattice defects, but also strain that leads to significant bowing of the layer-substrate stacks. ${ }^{14}$ 


\section{WILEY-VCH}

In general, the density of extended defects, mainly threading dislocations, and the concentration of point defects like impurity complexes or vacancies as well as the built-in electric fields are substantially governing the performance limits of $\mathrm{GaN}$ based components. Requirements for optoelectronic devices, amongst others, are a high radiative recombination efficiency, i.e. high internal quantum efficiency, and strong carrier injection into the active region. Both may be impaired by a high density of defects, either by current leakage and nonradiative recombination at threading dislocations or impurity complexes ${ }^{15}$ or by free carrier compensation through defects. ${ }^{16}$ Beyond this, the strong ionic character of GaN and the lack of inversion symmetry of its wurtzite lattice-structure lead to spontaneous and strain-related piezoelectric polarization fields in certain crystallographic orientations. Especially the mismatch-related strain in the heterostructure inside the active layers of LEDs introduces the quantum confined Stark effect which reduces the overlap of electron and hole wave functions inside the quantum well at low injection conditions and thus the probability for radiative recombination. ${ }^{17}$ On the other hand, these electric fields may be beneficial like in high electron mobility transistors (HEMTs) where they increase the electron concentration in the channel. ${ }^{18}$ However, detrimental phenomena like current collapse and off-state leakage in HEMTs have been shown to be closely related to extended defects. ${ }^{19}$ Therefore, high-quality GaN material with either polar, semipolar or nonpolar orientation for high-end electronic and optoelectronic devices is urgently needed.

Here we introduce GaN microfins as a suitable alternative platform for the application in such devices regarding the rare occurrence of extended defects as well as the availability of nonpolar (and semipolar) crystal planes, which may be capitalized upon in applications such as core-shell LEDs or vertical transistors. ${ }^{20,21,22}$ 


\section{WILEY-VCH}

\section{Experimental Section}

GaN fins have been fabricated by selective area growth on planar $\mathrm{SiO}_{x} / \mathrm{GaN} /$ sapphire templates. The $30 \mathrm{~nm}$ thick $\mathrm{SiO}_{\mathrm{x}}$ mask layer was structured by conventional photolithography. The line openings are aligned along the m-direction of the underlying GaN buffer layer in order to realize GaN fins with a-plane sidewalls. The mask openings are arranged in different patterns with various nominal widths (from $0.5 \mu \mathrm{m}$ to $2 \mu \mathrm{m}$ ) and pitches (from $1.5 \mu \mathrm{m}$ to 18 $\mu \mathrm{m})$. Accordingly, each pattern is named, e.g. W2 P18 for a nominal opening width of $2 \mu \mathrm{m}$ and an opening pitch of $18 \mu \mathrm{m}$. Using metalorganic vapor phase epitaxy (MOVPE) in a Thomas Swan close-coupled showerhead reactor with a 3 x 2" susceptor, GaN is selectively grown in the line openings of the mask, leading to the development of fin structures. Here, a continuous growth mode is used, in contrast to other publications, where a pulsed growth mode is applied to achieve vertical growth..$^{23,24}$ The growth process was optimized in order to achieve smooth sidewalls, smooth top surfaces as well as high homogeneity over the fin length and fin patterns. This optimized growth process, the 2-temperature-step-growth, was reported elsewhere. ${ }^{20}$ The most important parameter for a successful three-dimensional (3D) growth is a low $\mathrm{V} / \mathrm{III}$ ratio (here namely 77 ).

Subsequent to the MOVPE process, the fins are analyzed in a Tescan Mira3 GMH field emission scanning electron microscope (SEM). By the help of a customized Gatan MonoCL4 detection setup and a Kammrath \& Weiss C.80 liquid nitrogen cryo-stage, cathodoluminescence $(\mathrm{CL})$ measurements are performed at low and room temperature. CL spectra are obtained by a CCD parallel detector and corrected for the spectral responsivity of the detection system.

Scanning transmission electron microscopy (STEM) measurements are carried out using a JEOL JEM-2100F equipped with a bright-field (BF) and a high-angle annular dark-field (HAADF) detector. For the site-specific sample preparation, a JEOL JIB-4501 focused ion beam (FIB) device is employed. 


\section{WILEY-VCH}

\section{Threading dislocations}

One of the main advantages of 3D nanostructures is the reduced threading dislocation (TD) density in comparison to the underlying buffer layer. Several studies state that extended dislocations which propagate into the vertical nanostructure tend to bend towards the sidewalls during the initial stage of the growth so that the upper part of the 3D structures exhibits a strongly reduced dislocation density. ${ }^{25,26}$ STEM investigations (Figure 1 a) of a fin cross-section demonstrate that a few TDs are propagating into the fin and terminating not always close to the fin base, but rather at different heights at the sidewall. Some of them are even reaching through to the top facet. The TD density of the a-plane sidewall is estimated as the number of TD piercing through a certain a-plane surface area divided by the size of this area. The measurement is realized by preparing a sample for TEM investigation in a way that the a-plane sidewall is thinned from one side of the fin. Thereby, the growth surface of one side of the a-plane sidewalls remains undamaged and a TEM observation along the a-plane normal is possible. Electron transparency is obtained for a relatively large area of $56 \mu \mathrm{m}^{2}$ (Figure 1b). However, only a single TD is found within this area leading to an estimated TD density of $1.8 \times 10^{6} \mathrm{~cm}^{-2}$. Although a single measurement does not yield a statistically sound result, it provides a rough estimate on the TD density, which is drastically reduced as compared to the underlying buffer layer $\left(8 \times 10^{8} \mathrm{~cm}^{-2}\right)$. To explain this behavior, several studies on dislocation evolution from publications on nanorods are considered, stating that the position of the TDs plays an important role. Particularly, in 1953 Eshelby established that screw dislocations located off-center at a distance of more than 0.54 of the radius, would bend towards the sidewalls as a result of image forces. ${ }^{27}$ On the contrary, the symmetric forces from opposing surface facets of a rod would keep the dislocation in the center along its axis. Although the value given of 0.54 would differ for the GaN fins due to their geometry and 


\section{WILEY-VCH}

their piezoelectric properties, the principle explanation is assumed to be transferable. Since

the mask openings are nominally $2 \mu \mathrm{m}$ wide, while the final fin width is about $7 \mu \mathrm{m}$, the majority of the TDs would terminate on the c-plane and only the ones close to the rims would bend towards the sidewalls and terminate there, as observed in the TEM results. This results in a drastically reduced threading dislocation density in the sidewall planes.
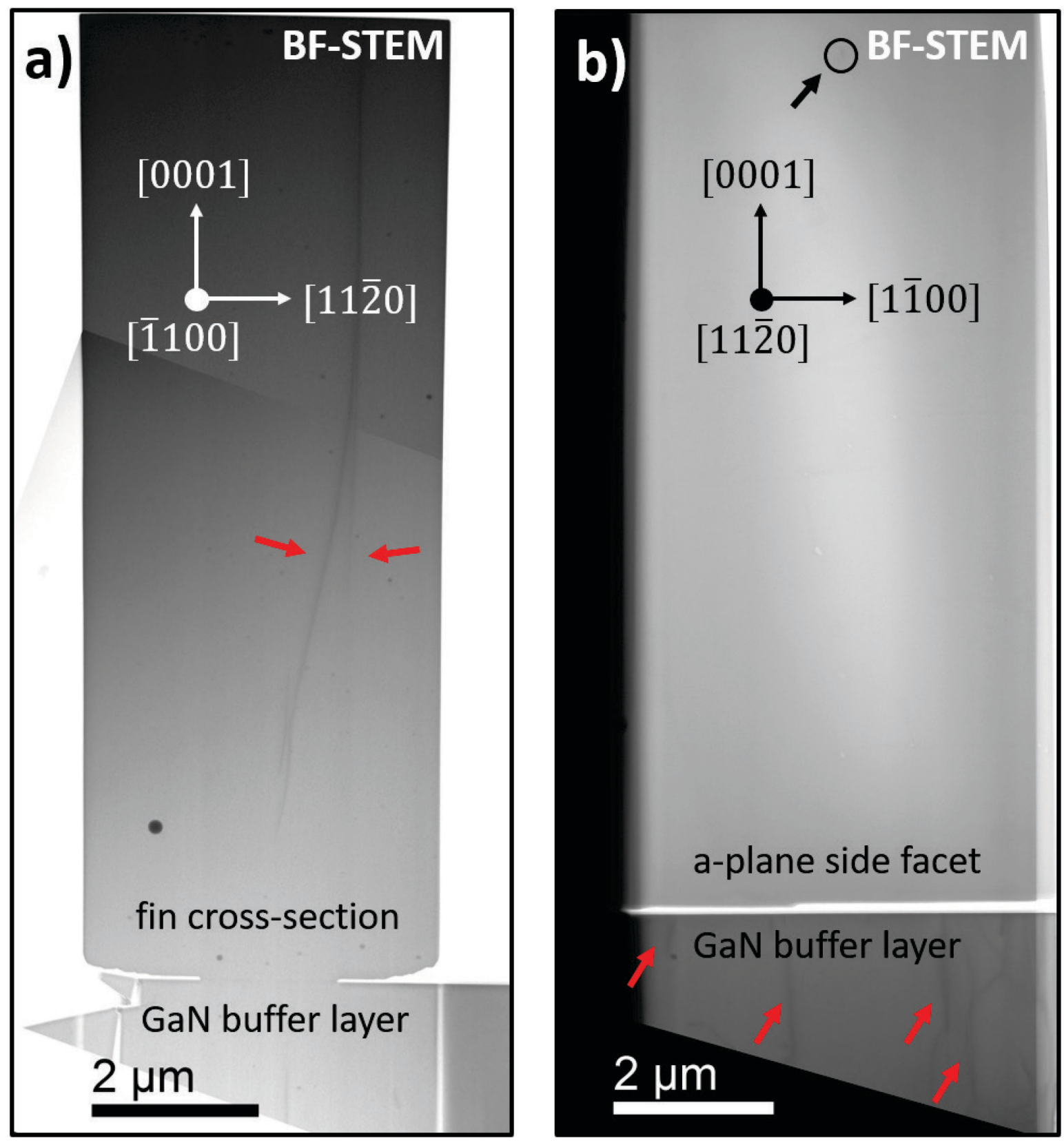

Figure 1. Cross-sectional BF-STEM micrographs of a fin viewing along the [1100] zone axis (a) and perpendicular to an a-plane sidewall along the [11̄20] zone axis (b). Dislocations are marked with arrows. Only a single dislocation pierces through the a-plane sidewall (see black arrow and circle). 


\section{WILEY-VCH}

Due to the size of the fins, as well as the time-consuming sample preparation for TEM, CL is additionally used to gain higher statistical relevance of the results. Since dislocations act as non-radiative sites, they may be identified by CL as dark spots on the surface where they terminate. ${ }^{28,29}$ The CL analysis is performed on fins from the pattern W2 P18 (grown with the 2-temperature-step-growth) and under liquid nitrogen temperatures to suppress non-radiative carrier recombination and thus enhance the contrast of dislocations. In accordance with TEM investigations giving an upper limit for the TD density of $1.6 \times 10^{6} \mathrm{~cm}^{-2}$, panchromatic CL maps (Figure $2 \mathrm{~b}$ ) show dislocations terminating on the sidewall with a density of $3 \times 10^{5} \mathrm{~cm}^{-2}$ (with a standard deviation of $2 \times 10^{5} \mathrm{~cm}^{-2}$ ) measured over a total fin sidewall area of 0.014 $\mathrm{mm}^{2}$. This TD density is three orders of magnitude lower than in the buffer layer $\left(8 \times 10^{8} \mathrm{~cm}^{-}\right.$ ${ }^{2}$ ) and competitive with bulk GaN substrates grown by HVPE. ${ }^{30}$ The c-plane CL efficiency of the fin cores is extremely poor due to the high concentration of impurities or intrinsic point defects in this growth direction and under the employed 3D growth parameters, which will be explained in the next section. In order to obtain a contrast in CL maps from this plane, and thus identify the TDs, a high-quality GaN shell layer is grown around the fin (shown in Figure $2 \mathrm{c}$ and $\mathrm{d}$ ). In contrast to the sidewalls, the top facet exhibits a larger dislocation density (c.f. room temperature CL map in Figure 2 d), consistent with the model of dislocation propagation introduced above. 


\section{WILEY-VCH}
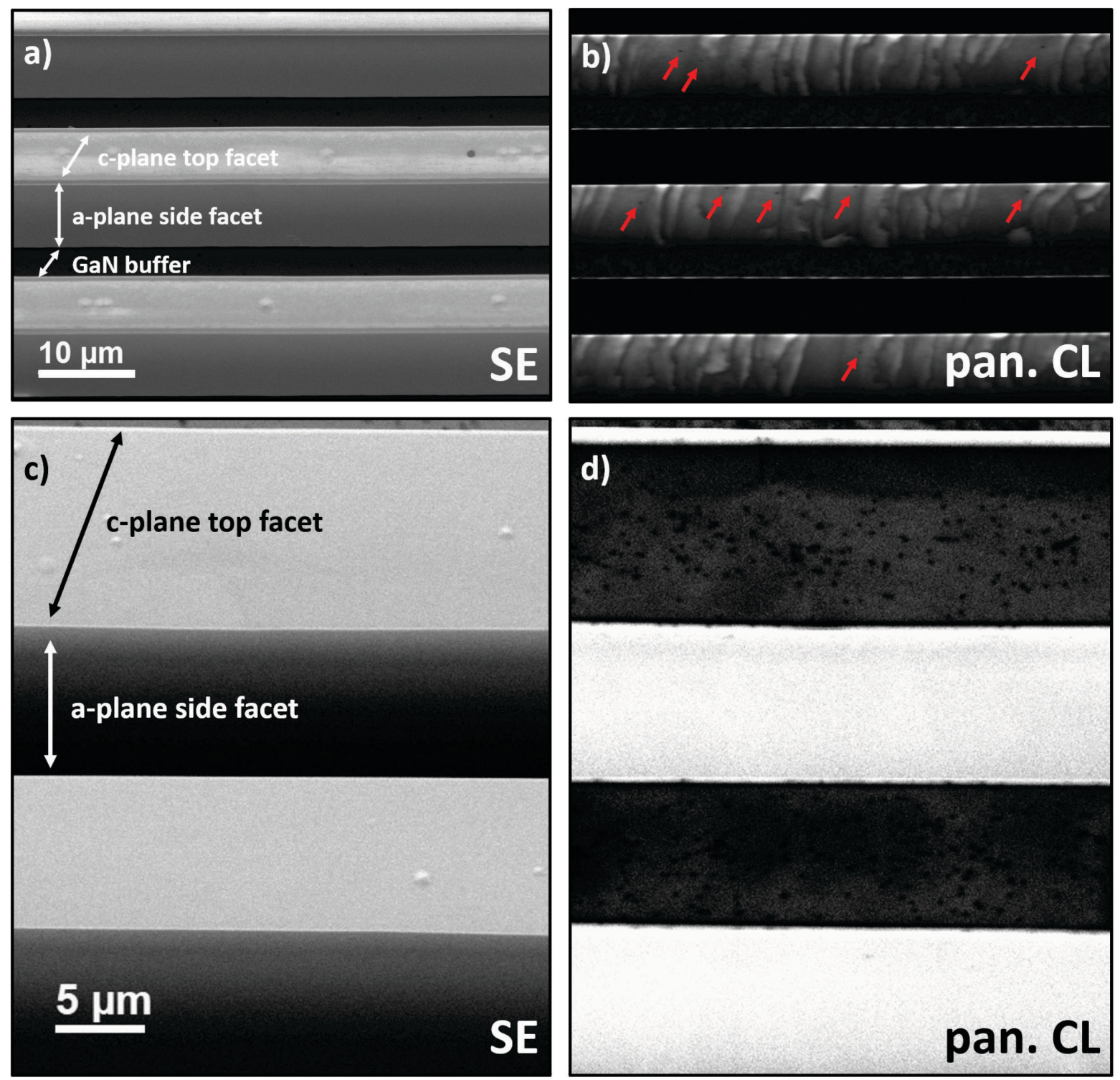

Figure 2. (a) SE map (5 keV, $30^{\circ}$ tilt) of three fins in pattern W2 P18. (b) panchromatic CL map of the same region, measured at liquid nitrogen temperature. Dark spots in the CL signal are marked with red arrows. Changes in the luminescence on the sidewalls could be related to step bunching as a result of imperfect alignment of the mask opening with the crystal's mdirection. (c) SE map of two fins covered by a high-quality GaN shell. (d) panchromatic CL map of the same region (signal clipped to enhance contrast on the c-plane top facet), measured at room temperature. 


\section{WILEY-VCH}

\section{Fin Growth Evolution}

A particularly unexpected feature of fin structures is the absence of near band edge (NBE) emission from the fin's top facet. In order to explore the underlying reasons, SEM and CL measurements from a cross-section of fins are analysed (cf. e.g. Figure 3). Two substantial features can be observed: Firstly, a contrast between the central region of the fin and the rim regions is visible in the secondary electron (SE) signal, which points to different charging behavior during SEM measurements and thus different conductivity. Secondly, the NBE emission occurs almost only in the triangular rim regions close to the sidewalls (CL map in Figure $3 \mathrm{~b}$ and blue spectrum in Figure $3 \mathrm{c}$ ). The central region of the fins shows emission mainly in a broad yellow luminescence (YL) defect band around a wavelength of $550 \mathrm{~nm}$, as visible in CL maps (not shown here) and CL spectra (orange spectrum in Figure $3 \mathrm{c}$ ). 


\section{WILEY-VCH}
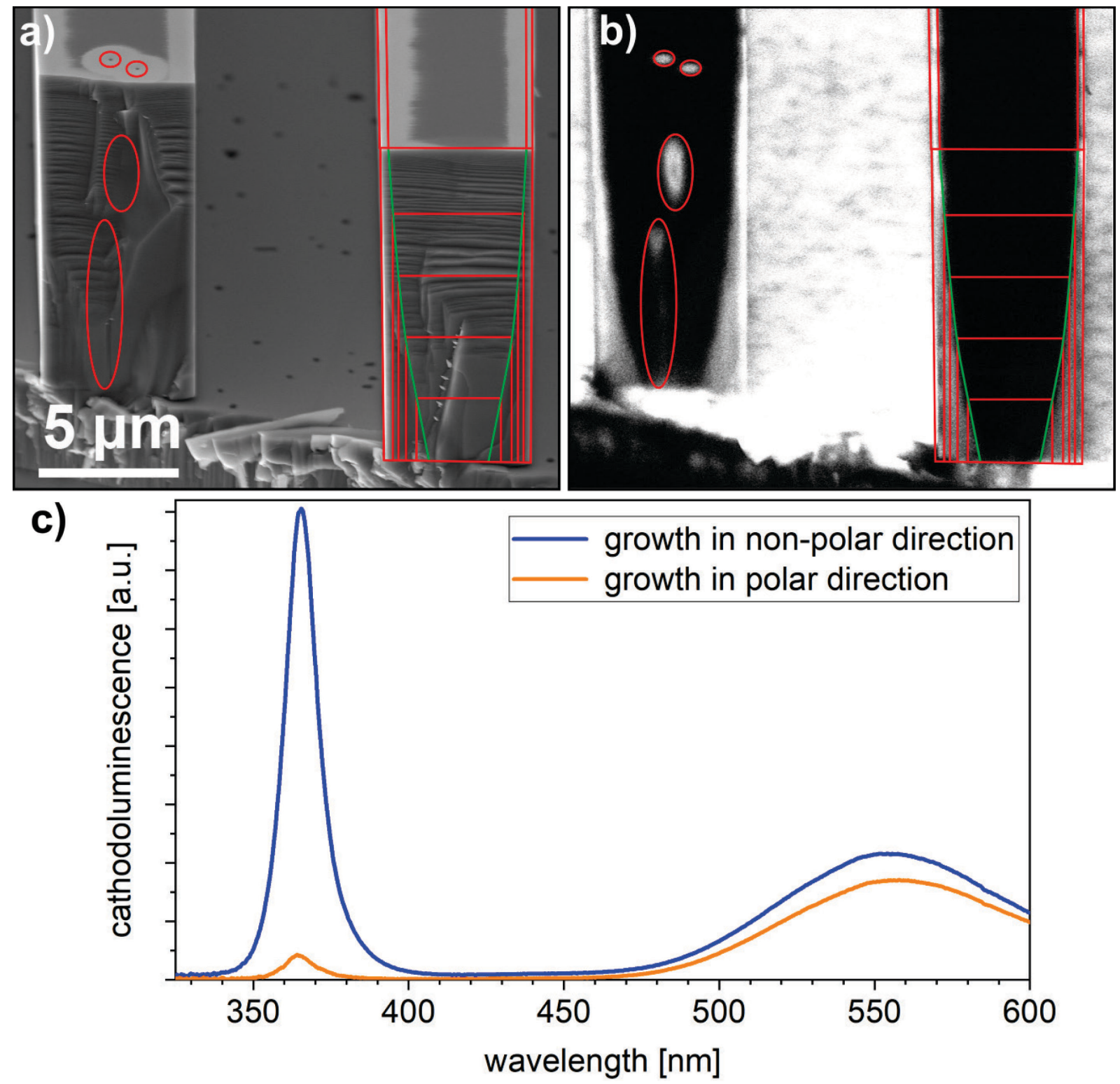

Figure 3. (a) SE map (10 keV, $60^{\circ}$ tilt) of fins in pattern W2 P12. (b) CL map at $365 \mathrm{~nm}$ wavelength of the same area. Vertical holes and the assumed growth fronts are marked in red in the left and right fin, respectively. ${ }^{31}$ (c) CL spectra from exciting the outer region (blue) and inner region (orange) of a cross-section of a similar fin.

To clarify this behavior and relate the properties of fins to their growth mechanism, an experiment introducing marker layers during growth is carried out. In this way, the evolution of the growth front during the epitaxy can be visualized at certain times without interrupting the growth process. ${ }^{32,33,34}$ Ten thin marker layers with elevated silicon doping (standard $\mathrm{SiH}_{4}$ flow during fin growth is $16.5 \mathrm{nmol} / \mathrm{min}$, during marker layer growth it is increased to 715 $\mathrm{nmol} / \mathrm{min}$ for $10 \mathrm{~s}$ ) are introduced. The marker layer parameters are adjusted in a way that on 


\section{WILEY-VCH}

the one hand, its influence on the subsequent 3D growth is minimal and on the other hand, the contrast in SE and CL signals is unambiguously detectable. The latter is the reason why the influence of the marker layer on the following growth steps can not be avoided completely. It was reported before that high silicon doping during 3D GaN growth can lead to enhanced vertical growth rates and a passivation of lateral surfaces..$^{35,36,37,38}$ This passivation effect is also detected on $\mathrm{GaN}$ fins with marker layers, where no further lateral growth occurs on the already grown a-plane sidewalls (Figure 4) and a higher growth rate of the subsequently growing a-facet due to an increased supply of growth species above the passivated parts is expected. This is the reason why the fins with marker layers do not have smooth a-plane sidewalls, but a step-like surface (Figure 4 a) and also exhibit a semipolar growth front. Despite this perturbation of the growth process, some conclusions can be drawn from these marker-layer fin-like architectures. The highly silicon-doped marker layers are visible in inbeam-SE maps (Figure 4 b) as well as in CL maps. They exhibit the three main growth directions: the polar c-direction $\{0001\}$, the semipolar directions (mostly in $\{11 \overline{2} 2\}$, but not exclusively) and the nonpolar $\{11 \overline{2} 0\}$ directions. The distance between marker-layers can be used as a measure for the growth rate in the respective direction (arrows in Figure 4 b) but it needs to be noted that the growth rates may differ from those in structures grown without marker-layers. Since there is no further growth on the a-plane after the marker-layer growthstep, as discussed above, some species may diffuse along the sidewall and be incorporated on the a-plane above the passivated region. Thus, it is assumed that the growth velocity in the nonpolar $\{11 \overline{2} 0\}$ directions is enhanced. This growth rate increase could lead to the appearance of the semipolar $\{11 \overline{2} 2\}$ facets, which are not visible in most fins grown under standard growth conditions without marker layers. In these standard fins, the semipolar facet was only detected during the first stages of growth. 


\section{WILEY-VCH}

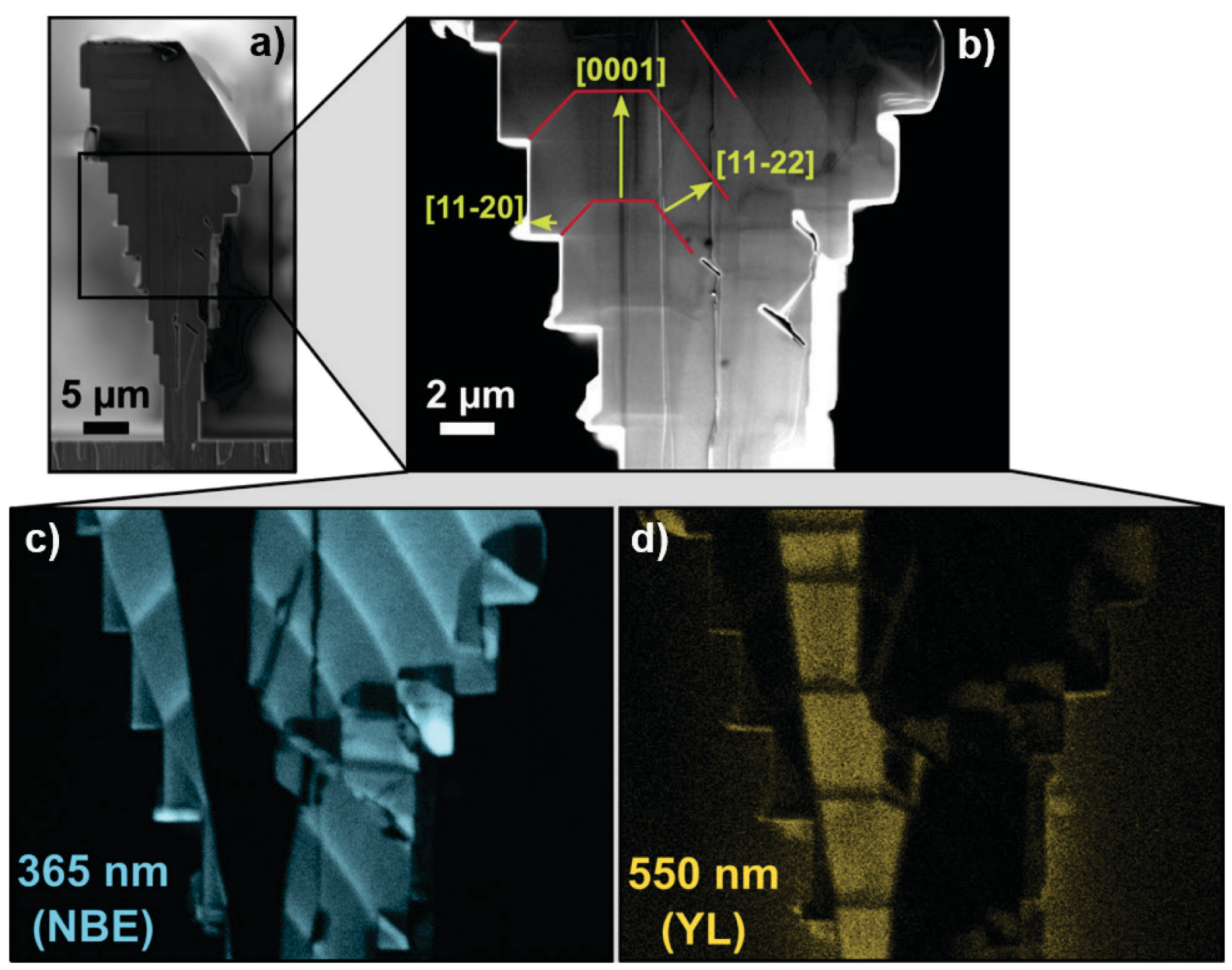

Figure 4. (a) SE map (5 keV) of the cross-section of a fin-like structure with ten highly doped marker layers along the height. (b) Higher magnification in-beam-SE map (5 keV) of the cross-section of the same fin-like structure. Colored bandpass CL maps at $365 \mathrm{~nm}$ (c) and 550 $\mathrm{nm}$ wavelength $(\mathrm{d})$ of the same magnified region as in (b). ${ }^{31}$

The relative growth velocities, evaluated based on several in-beam-SE maps of marker layer

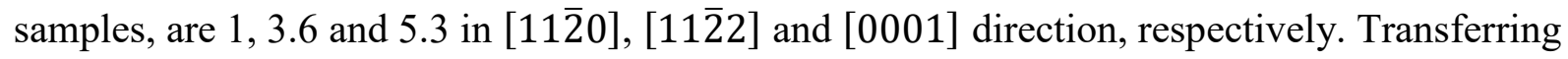
these results to the growth of regular fins without highly doped marker-layers, it is assumed that no passivation on the sidewalls occurs due to the constantly low $\mathrm{SiH}_{4}$ flow during epitaxy. The relative growth velocities in the three directions can be visualized by arrows as shown in Figure 5 a. After the first growth step, the growth fronts are drawn in Figure 5 b. Since the growth velocity in the nonpolar directions is very slow and a factor of 5.8 to 8 faster in polar direction (cf. right fin in Figure 3: In the lower part of the fin, where the diffusing species from the adjacent $\mathrm{SiO}_{\mathrm{x}}$ mask are incorporated on a still small a-facet, this ratio is 5.8, 


\section{WILEY-VCH}

whereas in the upper part of the fin, where the influence of the masked area is less distinct, the ratio is 8), the semipolar planes do not develop at all, as shown schematically by the results from the Wulff construction ${ }^{39}$ in Figure $5 \mathrm{~b}$ and c. Only nonpolar and polar planes would determine the final shape of the fin (Figure $5 \mathrm{c}$ and $\mathrm{d}$ ). This is exactly what is found for standard fin growth ${ }^{20}$, where the semipolar facet appears only in the initial stages of growth. Thus, the growth model with the relative growth velocities, derived from the marker-layer experiments, can be assumed to pertain for the MOVPE conditions applied here.

a)

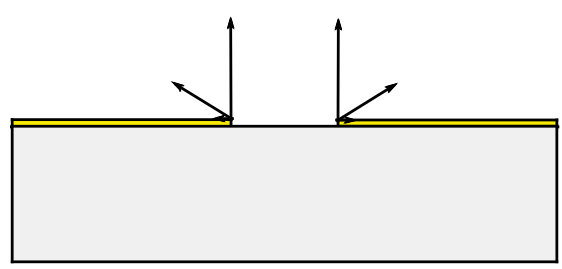

c)

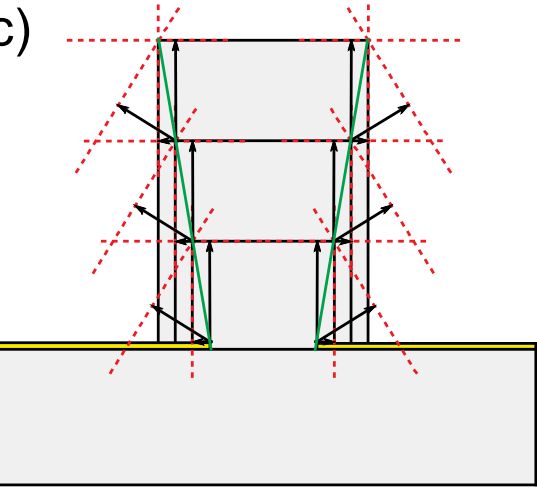

b)
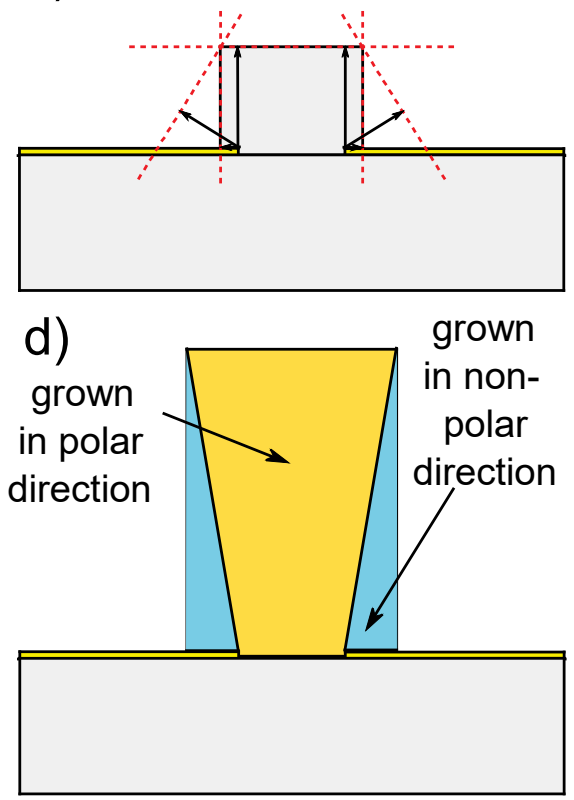

Figure 5. Model of SAG of fins with the relative growth velocities estimated from markerlayer growth experiments: (a) Mask opening with growth starting at the rim, evolving planes after short (b) and long (c) growth time, and (d) resulting transition between inner region and outer triangular regions. ${ }^{31}$

\section{Impurities}

Analyzing the fin-like structures grown with marker-layers by CL, mainly GaN grown in the polar direction shows a defect-related YL around $550 \mathrm{~nm}$, as visible in Figure 4 d. However, GaN grown in the semipolar directions does not show intensive YL, but mainly NBE emission at $365 \mathrm{~nm}$ (Figure $4 \mathrm{c}$ ). GaN grown in the nonpolar directions also shows mainly NBE emission and a slightly higher YL. 


\section{WILEY-VCH}

Transferring these findings to the growth without marker-layers reveals two regions in the core of most fins: the inner region grown in polar direction and the outer region grown in nonpolar direction (Figure $5 \mathrm{~d}$ ). As already discussed in section 4, the inner region shows low CL dominated by YL while the outer regions with a triangular shape in the cross-section exhibit intense NBE emission. This is observed for fins grown under standard conditions, as e.g. with the 2-temperature-step-growth (not shown here). ${ }^{20}$

In general, a YL band is not unexpected in 3D structures since their growth conditions favor the incorporation of point defects and impurities by the low V/III ratio during epitaxy required for high aspect ratios. The incorporation may additionally depend on the orientation of growth. Cruz et al. analyzed this for the most interesting impurities and dopants in MOVPE grown $\mathrm{GaN}$ on differently oriented substrates. ${ }^{40}$ By using low V/III ratio, i.e. similar to fin epitaxy, they found more than one order of magnitude more carbon in [0001] grown GaN than in layers grown in [112̄0] direction, whereas oxygen and silicon are more or less isotropically incorporated. However, it was reported that silicon incorporation is reciprocally proportional to the GaN growth rate. ${ }^{41}$ Thus, we expect a higher silicon concentration in the triangular side regions due to the slower growth rate in a-direction and a higher carbon content in the central part of the fins' cross-section due to growth in c-direction and low V/III ratio. The higher carbon concentration leads to an increased YL intensity ${ }^{42}$ which would explain the contrast in YL CL maps as in Figure $4 \mathrm{~d}$. Additionally, the central fin regions grown in c-direction exhibit more charging effects during scanning with the electron beam compared to the outer regions (see top facets of fins in Figure 3 a). Electrical characterization by a probe tip contacting along the cross-section of a fin reveals that this charging contrast in SE maps corresponds to an abrupt transition in electrical conductivity rather than to an insulating interface. This also agrees to the reduced silicon and increased carbon incorporation, which could correspond to compensation of background n-type conductivity by a high carbon concentration. ${ }^{16}$ Only around vertical holes in the inner region of fins, as 


\section{WILEY-VCH}

marked by red ovals in Figure 3 a and b, less charging and higher NBE are detected. This is consistent with the model, since it is found by the marker-layer based fin-like structures that material around vertical holes is mainly grown in the semipolar direction and would thus incorporate less intrinsic defects or carbon impurities and would be better conductive.

Our hypothesis that different concentrations of silicon and carbon impurities are incorporated due to the extremely low V/III ratio needed for continuous-mode selective area epitaxy needs to be further corroborated. For this reason, secondary ion mass spectroscopy (SIMS) is employed to measure the distributions of silicon and gallium across a GaN fin detached from its growth template and placed on a foreign substrate with the a-plane sidewall facing upwards. In order not to interfere with the measurement, a germanium substrate is chosen. The acquired depth profiles of gallium and silicon, the latter scaled to atomic concentration in $\mathrm{GaN}$, are depicted in Figure 6 with a schematic of where they were acquired on the fin (hatched area). While the gallium signal yields an indication to scale the ablation crater in the GaN fin, the Si signal shows two clearly different regions, which, according to our previously introduced growth model, can be attributed to material grown in nonpolar or polar direction. The initially probed region grown in the nonpolar a-direction is found to incorporate a higher concentration of silicon, $2 \times 10^{19} \mathrm{~cm}^{-3}$, as opposed to the material grown in polar c-direction with a concentration of $3 \times 10^{18} \mathrm{~cm}^{-3}$. The lower slope of the Si increase at the backside of the lying fin (starting at approx. $2 \mu \mathrm{m}$ etching depth) could probably be explained by a rougher bottom of the etching crater and thus a blurred transition from polar grown to nonpolar grown material (which is also reflected by the slope of the Ga signal). Interestingly, the ratio of 6.7 between silicon concentrations in material grown in a- and c-directions roughly corresponds to the inverse ratio of growth rates of 5.8 to 8 , as explained in section 4 . Hence, it could be speculated that, amongst plane-independent incorporation efficiencies of silicon ${ }^{40}$, a lower $\mathrm{GaN}$ growth rate leads to a higher silicon concentration. ${ }^{41}$ 


\section{WILEY-VCH}

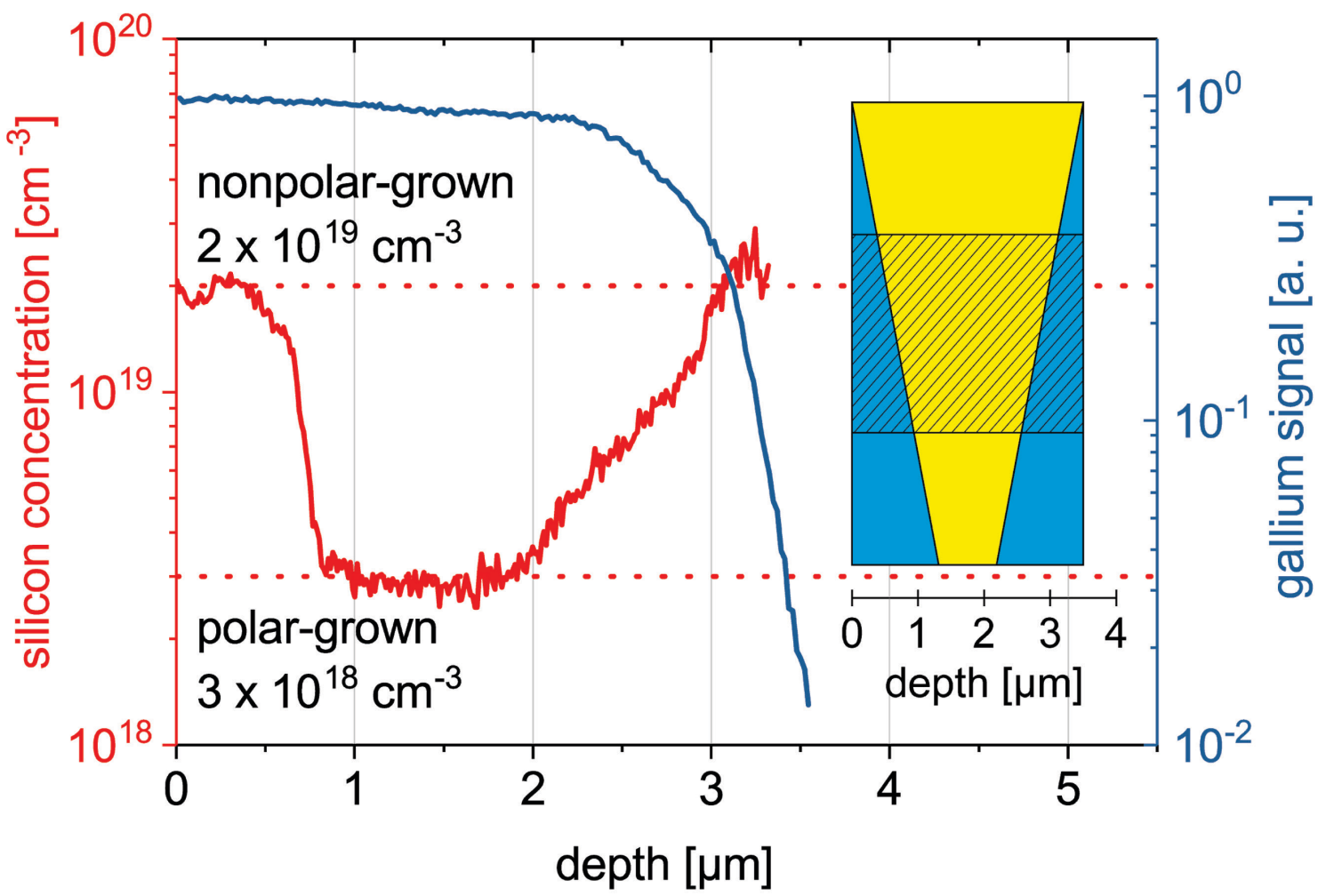

Figure 6. SIMS depth profile of gallium and silicon of a detached GaN fin placed on a germanium substrate with its a-plane sidewall facing upwards. The examined region of the fin is depicted in the accompanying schematic.

Since SIMS only provides information on the concentration of atoms present in a sample and not about the electrical activity of dopants, the SIMS measurement is contrasted with a local measurement of the carrier concentration using scanning capacitance microscopy (SCM) on a cleaved fin cross-section. A detailed report on locally resolved carrier concentrations in $\mathrm{GaN}$ fins correlating SCM and $\mu$ Raman spectroscopy measurements is currently in preparation. As a result, in the outer regions of the fin, which are grown in nonpolar direction, an electron concentration comparable to a planar high-quality GaN sample with a silicon concentration of $(2 \pm 1) \times 10^{19} \mathrm{~cm}^{-3}$ is obtained. In the inner part grown in polar direction, however, no free carriers above the instrument's sensitivity of $1 \times 10^{15} \mathrm{~cm}^{-3}$ could be detected. Thus, electrons originating from the silicon donors present in this region are heavily trapped. Carbon impurities are the most probable cause for carrier compensation in n-doped GaN. ${ }^{16}$ Alongside the observation of strong quenching of NBE emission in favor of a YL band in CL, the 


\section{WILEY-VCH}

occurrence of heavy carrier compensation clearly hints at an excessive carbon incorporation in the central region of GaN fins. In contrast to this, an enhanced silicon incorporation in the sidewall regions grown with lower growth velocities, e.g. in semipolar or nonpolar direction, in conjunction with an apparently much lower impurity incorporation leads to a high concentration of free electrons and strong NBE emission in these parts of GaN fins.

\section{Summary}

In this report, the suitability of GaN fins for the application in electronic and optoelectronic devices is examined with regard to the behavior of extended defects, i.e. threading dislocations, and impurity incorporation leading to compensation of free carriers. Transmission electron microscopy and cathodoluminescence mapping at cryogenic temperature independently confirm that dislocations penetrating from the buffer into the microstructure do not predominantly bend towards the sidewalls, but rather terminate at the top facet. The nonpolar fin sidewalls exhibit ultra-low threading dislocation densities in the $10^{5} \mathrm{~cm}^{-2}$ range, making them competitive with GaN bulk substrates. Furthermore, the incorporation of point defects into GaN fins is examined by cathodoluminescence, local electrical contacting inside a scanning electron microscope and a combination of secondary ion mass spectroscopy and a local measurement of the free carrier concentration using scanning capacitance microscopy. The observation of distinctly different regions within the cross-section of fins is explained in terms of a growth front model. The central region of the fin grows in the polar c-direction, while the sidewall regions are based on growth in nonpolar a-direction. Both, the strong quenching of near band edge emission and the occurrence of a pronounced yellow luminescence band as well as heavy compensation of free electrons in the central region strongly hint to excessive carbon impurity incorporation during selective area growth under low V/III ratios and in polar direction. In contrast, the sidewall regions grown in nonpolar direction exhibit strong near band edge emission and a high carrier concentration 


\section{WILEY-VCH}

alongside ultra-low threading dislocation density and the absence of spontaneous and piezoelectric polarization fields, making fins ideally suited for three-dimensional core-shell LEDs or vertical electronics.

\section{Acknowledgements}

We (J.H., H.Z., A. J., T.V., A.W.) would like to acknowledge the financial support of the DFG within the research unit FOR1616 (Dynamics and Interactions of Semiconductor Nanowires for Optoelectronics). Additionally, we (I.M.C., C.M., H.S., F.S., H.-H.W.) acknowledge financial support from the epitaxy competence center $\mathrm{ec}^{2}$ and from “Niedersächsisches Vorab” through “Quantum- and Nano- Metrology (QUANOMET)” initiative within the project group NL2 (J.H.). The scholarship support by the China Scholarship Council is highly acknowledged (H.Z.). Moreover, we thank Juliane Breitfelder, Karl-Heinz Lachmund, Manfred Karsten and Andreas Heidemann for technical support and Margarita Matzeck for the TEM FIB-lamella preparation.

Received: ((will be filled in by the editorial staff))

Revised: ((will be filled in by the editorial staff)) Published online: ((will be filled in by the editorial staff))

References

(1) Wang, M.-T.; Liao, K.-Y.; Li, Y.-L. IEEE Photonics Technol. Lett. 2011, 23 (14), 962964.

(2) Oh, T. S.; Jeong, H.; Lee, Y. S.; Seo, T. H.; Park, A. H.; Kim, H.; Lee, K. J.; Jeong, M. S.; Suh, E.-K. Thin Solid Films 2011, 519 (8), 2398-2401.

(3) Preble, E. A.; Leach, J. H.; Metzger, R.; Shishkin, E.; Udwary, K. A. Phys. status solidi 2014, $11(3-4), 604-607$.

(4) Fritze, S.; Drechsel, P.; Stauss, P.; Rode, P.; Markurt, T.; Schulz, T.; Albrecht, M.;

Bläsing, J.; Dadgar, A.; Krost, A. J. Appl. Phys. 2012, 111 (12), 124505. 


\section{WILEY-VCH}

(5) Wang, W.; Yang, H.; Li, G. J. Mater. Chem. C 2013, 1 (26), 4070.

(6) Li, G.; Wang, W.; Yang, W.; Wang, H. Surf. Sci. Rep. 2015, 70 (3), 380-423.

(7) Tian, P.; Edwards, P. R.; Wallace, M. J.; Martin, R. W.; McKendry, J. J. D.; Gu, E.;

Dawson, M. D.; Qiu, Z. J.; Jia, C.; Chen, Z.; Zhang, G.; Zheng, L.; Liu, R. J. Phys. D. Appl. Phys. 2017, 50 (7).

(8) Dang, S.; Li, C.; Lu, M.; Guo, H.; He, Z. Optik (Stuttg). 2018, 155, 26-30.

(9) Mukai, T.; Morita, D.; Nakamura, S. J. Cryst. Growth 1998, 189-190, 778-781.

(10) Kneissl, M.; Kolbe, T.; Chua, C.; Kueller, V.; Lobo, N.; Stellmach, J.; Knauer, A.;

Rodriguez, H.; Einfeldt, S.; Yang, Z.; Johnson, N. M.; Weyers, M. Semicond. Sci.

Technol. 2011, $26(1), 014036$.

(11) Hirayama, H.; Maeda, N.; Fujikawa, S.; Toyoda, S.; Kamata, N. Jpn. J. Appl. Phys. 2014, 53 (10), 100209.

(12) Imanishi, M.; Todoroki, Y.; Murakami, K.; Matsuo, D.; Imabayashi, H.; Takazawa, H.; Maruyama, M.; Imade, M.; Yoshimura, M.; Mori, Y. J. Cryst. Growth 2015, 427, 8793.

(13) YOLE DEVELOPPEMENT FOR IMMEDIATE RELEASE : Bulk GaN : from technology to market http://www.yole.fr/iso_upload/News/2017/PR_BULKGAN_Applications_Players_YO LE_Feb2017.pdf.

(14) Li, G.; Wang, W.; Yang, W.; Lin, Y.; Wang, H.; Lin, Z.; Zhou, S. Reports Prog. Phys. 2016, $79(5), 056501$.

(15) Karpov, S. Y.; Makarov, Y. N. Appl. Phys. Lett. 2002, 81 (25), 4721-4723.

(16) Armstrong, A.; Arehart, A. R.; Moran, B.; DenBaars, S. P.; Mishra, U. K.; Speck, J. S.; Ringel, S. A. Appl. Phys. Lett. 2004, 84 (3), 374-376.

(17) Verma, J.; Verma, A.; Protasenko, V.; Islam, S. M.; Jena, D. In Nitride Semiconductor Light-Emitting Diodes (LEDs); Elsevier, 2014; pp 368-408. 


\section{WILEY-VCH}

(18) Hashizume, T.; Nishiguchi, K.; Kaneki, S.; Kuzmik, J.; Yatabe, Z. Mater. Sci. Semicond. Process. 2018, 78, 85-95.

(19) Ghosh, S.; Das, S.; Dinara, S. M.; Bag, A.; Chakraborty, A.; Mukhopadhyay, P.; Jana, S. K.; Biswas, D. IEEE Trans. Electron Devices 2018, 65 (4), 1333-1339.

(20) Hartmann, J.; Steib, F.; Zhou, H.; Ledig, J.; Nicolai, L.; Fündling, S.; Schimpke, T.; Avramescu, A.; Varghese, T.; Trampert, A.; Straßburg, M.; Lugauer, H.-J.; Wehmann, H.-H.; Waag, A. J. Cryst. Growth 2017, 476.

(21) Li, S.; Waag, A. J. Appl. Phys. 2012, 111 (7), 071101.

(22) Yadav, C.; Kushwaha, P.; Khandelwal, S.; Duarte, J. P.; Chauhan, Y. S.; Hu, C. IEEE Electron Device Lett. 2014, 35 (6), 612-614.

(23) Yeh, T.-W.; Lin, Y.-T.; Ahn, B.; Stewart, L. S.; Daniel Dapkus, P.; Nutt, S. R. Appl. Phys. Lett. 2012, 100 (3), 033119.

(24) Rishinaramangalam, A. K.; Ul Masabih, S. M.; Fairchild, M. N.; Wright, J. B.; Shima, D. M.; Balakrishnan, G.; Brener, I.; Brueck, S. R. J.; Feezell, D. F. J. Electron. Mater. 2015, 44 (5), 1255-1262.

(25) Hersee, S. D.; Rishinaramangalam, A. K.; Fairchild, M. N.; Zhang, L.; Varangis, P. J. Mater. Res. 2011, 26 (17), 2293-2298.

(26) Colby, R.; Liang, Z.; Wildeson, I. H.; Ewoldt, D. A.; Sands, T. D.; García, R. E.; Stach, E. A. Nano Lett. 2010, $10(5), 1568-1573$.

(27) Eshelby, J. D. J. Appl. Phys. 1953, 24 (2), 176-179.

(28) Rosner, S. J.; Carr, E. C.; Ludowise, M. J.; Girolami, G.; Erikson, H. I. Appl. Phys. Lett. 1997, 70 (4), 420-422.

(29) Ledig, J.; Steib, F.; Hartmann, J.; Fündling, S.; Wehmann, H.-H.; Waag, A. In European Microscopy Congress 2016: Proceedings; Wiley-VCH Verlag GmbH \& Co. KGaA: Weinheim, Germany, 2016; pp 1043-1044.

(30) Goubara, S.; Matsubara, T.; Yukizane, K.; Arita, N.; Fujimoto, S.; Ezaki, T.; Inomoto, 


\section{WILEY-VCH}

R.; Yamane, K.; Okada, N.; Tadatomo, K. J. Cryst. Growth 2017, 478, 123-128.

(31) Hartmann, J. MOVPE selective area growth of GaN/InGaN rod and fin core-shell LEDs, 2018.

(32) Schimpke, T.; Mandl, M.; Stoll, I.; Pohl-Klein, B.; Bichler, D.; Zwaschka, F.; StrubeKnyrim, J.; Huckenbeck, B.; Max, B.; MÃ¹/4ller, M.; Veit, P.; Bertram, F.; Christen, J.; Hartmann, J.; Waag, A.; Lugauer, H.-J.; Strassburg, M. Phys. Status Solidi Appl. Mater. Sci. 2016, 213 (6).

(33) Tu, C.-G.; Su, C.-Y.; Liao, C.-H.; Hsieh, C.; Yao, Y.-F.; Chen, H.-T.; Lin, C.-H.; Weng, C.-M.; Kiang, Y.-W.; Yang, C. C. Nanotechnology 2016, 27 (2), 055302.

(34) Caliebe, M.; Han, Y.; Hocker, M.; Meisch, T.; Humphreys, C.; Thonke, K.; Scholz, F. 2016, $53(1), 46-53$.

(35) Haffouz, S.; Beaumont, B.; Gibart, P. MRS Internet J. Nitride Semicond. Res. 1998, 30 (8), e8.

(36) Koester, R.; Hwang, J. S.; Durand, C.; Dang, D. L. S.; Eymery, J. Nanotechnology 2010, $21(1), 015602$.

(37) Tessarek, C.; Heilmann, M.; Butzen, E.; Haab, a.; Hardtdegen, H.; Dieker, C.; Spiecker, E.; Christiansen, S. Cryst. Growth Des. 2014, 14 (3), 1486-1492.

(38) Hartmann, J.; Wang, X.; Schuhmann, H.; Dziony, W.; Caccamo, L.; Ledig, J.; Mohajerani, M. S.; Schimpke, T.; B??hr, M.; Lilienkamp, G.; Daum, W.; Seibt, M.; Stra??burg, M.; Wehmann, H. H.; Waag, A. Phys. Status Solidi Appl. Mater. Sci. 2015, $212(12), 2830-2836$.

(39) Laue, M. v. Zeitschrift für Krist. - Cryst. Mater. 1943, 105 (1-6).

(40) Cruz, S. C.; Keller, S.; Mates, T. E.; Mishra, U. K.; DenBaars, S. P. J. Cryst. Growth 2009, $311(15), 3817-3823$.

(41) Koleske, D. .; Wickenden, A. .; Henry, R. .; Twigg, M. . J. Cryst. Growth 2002, 242 (1-2), 55-69. 
WILEY-VCH

(42) Xu, S.; Hao, Y.; Zhang, J.; Jiang, T.; Yang, L.; Lu, X.; Lin, Z. 2013, 3654-3657. 


\section{WILEY-VCH}

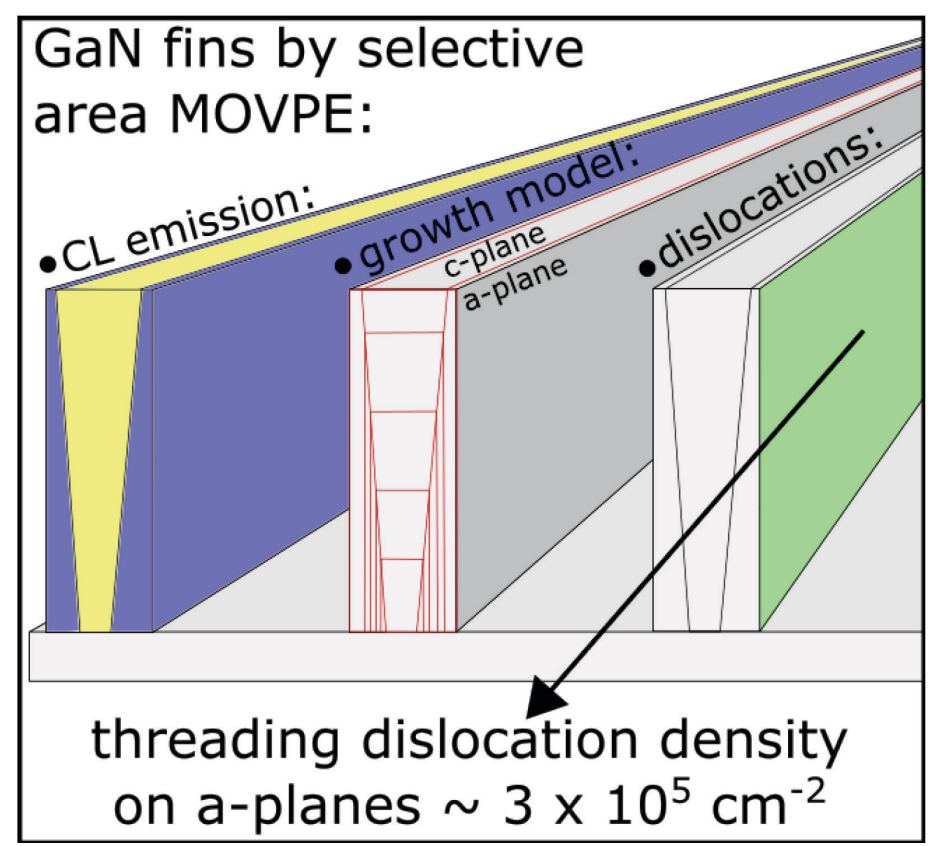

Copyright WILEY-VCH Verlag GmbH \& Co. KGaA, 69469 Weinheim, Germany, 2016. 